\title{
Effects of storage temperature, atmosphere and light on chemical stability of astaxanthin nanodispersions.
}

\begin{abstract}
Astaxanthin, as a functional lipid, can be incorporated easily into different water-based food formulations in the form of a nanodispersion. In this study, astaxanthin nanodispersions were produced using different stabilizer systems, namely, polysorbate 20 (PS20), sodium caseinate (SC), gum arabic (GA) and an optimum combination of these three stabilizers (OPT). Since astaxanthin is sensitive to oxidative damage, its degradations kinetics in the prepared nanodispersion systems were investigated as a function of storage temperature, atmosphere and light. The results showed that astaxanthin degradation followed a first-order kinetic and, in most cases, astaxanthin was more stable in optimum-formulated three-componentstabilized nanodispersions as compared to nanodispersion systems stabilized by individual stabilizers. In addition, high storage temperature and intense illumination significantly $(\mathrm{P}<$ $0.05)$ increased the degradation of astaxanthin, while oxygen-free conditions significantly ( $P$ $<0.05)$ reduced the astaxanthin degradation rate.
\end{abstract}

Keyword: Astaxanthin nanodispersion; Chemical stability; Emulsification-diffusion technique. 